\title{
О ПРИМИТИВНЫХ ГРУППАХ ПОДСТАНОВОК СО СТАБИЛИЗАТОРОМ ДВУХ ТОЧЕК, НОРМАЛЬНЫМ В СТАБИЛИЗАТОРЕ ОДНОЙ ИЗ НИХ: СЛУЧАЙ, КОГДА ЦОКОЛЬ ЕСТЬ СТЕПЕНЬ ГРУППЫ $E_{8}(q)$
}

\section{А. В. Коныгин}

\begin{abstract}
Пусть $G$ - примитивная группа подстановок на конечном множестве $X, x \in X, y \in X \backslash\{x\}$ и $G_{x, y} \unlhd G_{x}$. П. Камероном был поставлен вопрос о справедливости в этом случае равенства $G_{x, y}=1$. Ранее автором было доказано, что если цоколь группы $G$ не является степенью группы, изоморфной $E_{8}(q), q-$ степень простого числа, то $G_{x, y}=1$. В настоящей работе рассматривается случай, когда цоколь группы $G$ является степенью группы, изоморфной $E_{8}(q)$. Вместе с предыдущим результатом мы получаем два следующих утверждения: 1 . Пусть $G$ - почти простая примитивная группа подстановок на конечном множестве $X$. Предположим, что в случае, если цоколь $G$ изоморфен $E_{8}(q)$, то $G_{x}$ для $x \in X$ не является подгруппой Боровика в группе $G$. Тогда для таких примитивных групп подстановок $G$ ответ на вопрос П. Камерона положителен. 2. Пусть $G$ - примитивная группа подстановок на конечном множестве $X$ со свойством $G \leq H \mathrm{wr} S_{m}$. Предположим, что в случае, если цоколь группы $H$ изоморфен $E_{8}(q)$, то стабилизатор точки в группе $H$ не является подгруппой Боровика в группе $H$. Тогда для таких примитивных групп подстановок $G$ ответ на вопрос П. Камерона также положителен.
\end{abstract}

Ключевые слова: примитивная группа подстановок, регулярная подорбита.

A. V. Konygin. On primitive permutation groups with the stabilizer of two points normal in the stabilizer of one of them: The case when the socle is a power of a group $E_{8}(q)$.

Assume that $G$ is a primitive permutation group on a finite set $X, x \in X \backslash\{x\}$, and $G_{x, y} \unlhd G_{x}$. P. Cameron raised the question about the validity of the equality $G_{x, y}=1$ in this case. The author proved earlier that, if the socle of $G$ is not a power of a group isomorphic to $E_{8}(q)$ for a prime power $q$, then $G_{x, y}=1$. In the present paper, we consider the case where the socle of $G$ is a power of a group isomorphic to $E_{8}(q)$. Together with the previous result, we establish the following two statements. 1. Let $G$ be an almost simple primitive permutation group on a finite set $X$. Assume that, if the socle of $G$ is isomorphic to $E_{8}(q)$, then $G_{x}$ for $x \in X$ is not the Borovik subgroup of $G$. Then the answer to Cameron's question for such primitive permutation groups is affirmative. 2. Let $G$ be a primitive permutation group on a finite set $X$ with the property $G \leq H$ wr $S_{m}$. Assume that, if the socle of $H$ is isomorphic to $E_{8}(q)$, then the stabilizer of a point in the group $H$ is not the Borovik subgroup of $H$. Then the answer to Cameron's question for such primitive permutation groups is also affirmative.

Keywords: primitive permutation group, regular suborbit.

MSC: 20B15, 20D06

DOI: $10.21538 / 0134-4889-2019-25-4-88-98$

\section{1. Введение}

П. Камероном был сформулирован следующий вопрос (см. [3] и [23, вопрос 9.69]). Пусть $G$ - примитивная группа подстановок на конечном множестве $X, x \in X, y \in X \backslash\{x\}$ u $G_{x}$ действует регулярно на $G_{x}$-орбите $G_{x}(y)$, содержащей точку у (т. е. индуцирует на $G_{x}(y)$ регулярную группу подстановок). Верно ли, что это действие точное, т. е. что $\left|G_{x}\right|=$ $\left|G_{x}(y)\right|$ ? Отметим, что вопрос о точности действия стабилизатора $G_{x}$ на регулярной подорбите $G_{x}(y)$ изучался и ранее (см. $\left.[9 ; 13 ; 14 ; 21 ; 24 ; 25]\right)$.

Ясно, что регулярность действия группы $G_{x}$ на $G_{x}(y)$ эквивалентна свойству $G_{x, y} \unlhd G_{x}$, а равенство $\left|G_{x}\right|=\left|G_{x}(y)\right|$ эквивалентно равенству $G_{x, y}=1$. Таким образом, вопрос П. Камерона эквивалентен вопросу о выполнении для произвольной примитивной группы подстановок $G$ на конечном множестве $X$ следующего свойства:

(Pr) если $x \in X, y \in X \backslash\{x\}$, то $G_{x, y} \unlhd G_{x}$ влечет $G_{x, y}=1$. 
Очевидно, вопрос П. Камерона эквивалентен также вопросу о выполнении для произвольной конечной группы $G$ следующего свойства:

$\left(\mathbf{P r}^{*}\right)$ если $M_{1}$ и $M_{2}$ - различные сопряженные максимальные подгруппы группы $G$, то $M_{1} \cap M_{2} \unlhd M_{1}$ влечет $M_{1} \cap M_{2} \unlhd G$.

Согласно теореме О'Нэна - Скотта (см. [15]) любая конечная примитивная группа подстановок подстановочно изоморфна группе одного из перечисленных ниже типов.

I. Примитивные группы с абелевой регулярной нормальной подгруппой.

II. Примитивные почти простые группы. Напомним, что группа $G$ называется почти простой, если группа $G$ изоморфна подгруппе из $\operatorname{Aut}(T)$, содержащей $\operatorname{Inn}(T)$, для некоторой конечной простой неабелевой группы $T$.

III. Примитивные группы с неабелевым непростым цоколем. Среди групп этого типа различают группы трех типов:

(a) (simple diagonal action). Пусть $S_{k}$ - симметрическая группа степени $k \geq 2, T$ - простая неабелева группа и $W=\left\{\pi\left(a_{1}, \ldots, a_{k}\right) \mid a_{i} \in \operatorname{Aut}(T), \pi \in S_{k}, a_{i} a_{j}^{-1} \in \operatorname{Inn}(T), i, j \in\right.$ $\{1, \ldots, k\}\} \leq \operatorname{Aut}(T) w r S_{k}$. Тогда представление группы $W$ левыми сдвигами на множестве левых смежных классов группы $W$ по подгруппе $W_{x}=\left\{\pi(a, \ldots, a) \mid a \in \operatorname{Aut}(T), \pi \in S_{k}\right\}$ является точным примитивным представлением степени $|T|^{k-1}$. Конечная примитивная группа $G$ имеет тип III(a), если она изоморфна подгруппе группы $W$ в этом представлении, содержащей $\operatorname{soc}(W)$.

(b) (product action). Пусть $S_{m}$ - симметрическая группа степени $m \geq 2$ и $H$ - примитивная группа типа II или III(a) на конечном множестве $Y$. Положим $W=H \mathrm{wr} S_{m}$. Группа $W$ естественным образом действует на $X=Y^{m}$. Конечная примитивная группа $G$ имеет тип III(b), если она изоморфна подгруппе группы $W$ в этом представлении, содержащей $K^{m}$, где $K=\operatorname{soc}(H)$, и $G$ транзитивно переставляет $m$ прямых множителей группы $K^{m}$.

(c) (twisted wreath action). Конечная примитивная группа $G$ имеет тип III(c), если она обладает единственной неабелевой регулярной нормальной подгруппой.

Ранее в работе автора ( $\mathrm{K}$ вопросу Камерона о тривиальности в примитивных группах подстановок стабилизатора двух точек. Тр. Ин-та математики и механики УрО РАН. 2015. Т. 21, № 3. С. 175-186) было доказано, что если $G$ - примитивная группа подстановок на конечном множестве $X$ и либо $G$ - группа типа I, III(а) или III(c), либо $G$ - группа типа II с цоколем, не изоморфным $E_{8}(q), q$ - степень простого числа, то для группы подстановок $G$ выполняется свойство (Pr). Там же доказано, что если $G \leq H \mathrm{wr} S_{m}$ - группа типа III(b) и цоколь группы $H$ не изоморфен $E_{8}(q)$, то для группы подстановок $G$ также выполняется свойство $(\mathbf{P r})$. В частности, для всех таких примитивных групп подстановок $G$ ответ на вопрос П. Камерона положителен. В настоящей работе рассматривается случай, когда цоколь группы $G$ является степенью группы, изоморфной $E_{8}(q)$. С учетом предыдущих результатов автора доказываются следующие две теоремы.

Теорема 1. Пусть $G$ - почти простая примитивная группа подстановок на конечном множестве $X$. Предположим, что в случае, если чоколь $G$ изоморфен $E_{8}(q)$, то $G_{x} \partial л я x \in X$ не является подгруппой Боровика (см. предложение 1.IV ниже) в группе G. Тогда для группь подстановок $G$ выполняется свойство (Pr). В частности, для таких примитивных групп подстановок $G$ ответ на вопрос П. Камерона положителен.

Теорема 2. Пусть $G$ - примитивная группа подстановок на конечном множестве $X$ такал, что $G \leq H \mathrm{wr} S_{m}$ - группа типа III(b). Предположим, что если иоколь группы $H$ изоморфен $E_{8}(q)$, то стабилизатор точки в группе $H$ не является подгруппой Боровика (см. предложение $1 . \mathrm{IV}$ ниже) в группе $H$. Тогда для группы подстановок $G$ выполняется свойство (Pr). В частности, для таких примитивных групп подстановок $G$ ответ на вопрос П. Камерона положителен. 


\section{2. Обозначения и вспомогательные результаты}

Для произвольной конечной группы $G$ и простого числа $p$ в работе используются следующие стандартные обозначения: $\operatorname{soc}(G)$ - цоколь группы $G, F(G)$ - подгруппа Фиттинга группы $G, F^{*}(G)$ - обобщенная подгруппа Фиттинга группы $G$. Для групп $A$ и $B$ через $A . B$ будет обозначаться (см. [5]) произвольная группа $G$ с нормальной подгруппой $H$ такой, что $H \cong A$ и $G / H \cong B$. При указании структуры группы через $p^{n}$ будет обозначаться (см. [5]) элементарная абелевая группа порядка $p^{n}$.

Пусть $A$ и $B$ - группы. Подгруппу $D$ прямого произведения групп $A$ и $B$ назовем диагональной, если $A \cap D=B \cap D=1$ и $A B$ совпадает с $D A$ или $D B$.

Пусть $R$ - такая простая алгебраическая группа над алгебраически замкнутым полем $K$ ненулевой характеристики $p$ и $F$ - такой эндоморфизм Стейнберга алгебраической группы $R$, что $L=R^{F}:=\{g \in R \mid F(g)=g\}$ является конечной простой группой, изоморфной $E_{8}(q)$, где $q=p^{m}$. Пусть $Q-$ конечная группа такая, что $F^{*}(Q)=L$. Группа $\operatorname{Aut}(L)$ порождается сопряжениями посредством элементов из $R^{F}$ и полевыми автоморфизмами группы $L$, причем все эти автоморфизмы группы $L$ продолжаются до автоморфизмов абстрактной группы $R$, коммутирующих с $F$. Таким образом, существует подгруппа $\tilde{Q}$ группы $C_{\mathrm{Aut}(R)}(F)$ такая, что $Q=\tilde{Q} /\langle F\rangle$, и, следовательно, $Q$ действует на множестве всех $F$-допустимых подмножеств группы $R$. Через $N_{Q}(V)$ будем обозначать нормализатор в $Q$ произвольного $F$-допустимого подмножества $V$ группы $R$. Если $D$ является $F$-допустимой замкнутой связной редуктивной подгруппой группы $R$, содержащей максимальный тор группы $R$, и $M=N_{Q}(D)$, то $M$ называется группой максимального ранга в $Q$. Через $\mathcal{L}(R)$ обозначается алгебра Ли группы $R$. Будем предполагать, что вершины диаграмм Дынкина пронумерованы стандартным образом (см. [22]).

Пусть $X-$ простая алгебраическая группа над алгебраически замкнутым полем $K$. Для доминантного веса $\lambda$ пусть $L_{X}(\lambda)$ - рациональный неприводимый $K X$-модуль старшего веса $\lambda$ и $T_{X}(\lambda)-$ неразложимый тилтинг-модуль (tilting module) старшего веса $\lambda$. Следуя [18;22], будем писать $L(\lambda)$ или $\lambda$ для обозначения модуля $L_{X}(\lambda)$ и $a_{1} \ldots a_{r}$ - вместо доминантного веса $\sum_{i=1}^{r} a_{i} \omega_{i}$, где $\omega_{i}-$ фундаментальные доминантные веса и $a_{1}, \ldots, a_{r}-$ неотрицательные целые числа (неоднозначность этих обозначений устраняется контекстом использования). Для $K X$-модулей $M_{i}, i \in\{1, \ldots, k\}$, через $M_{1}\left|M_{2}\right| \ldots \mid M_{k}$ будем обозначать рациональный $K X$-модуль $V$, имеющий такой ряд подмодулей $0=V_{k}<V_{k-1}<\ldots<V_{1}<V_{0}=V$, что $V_{i-1} / V_{i} \cong M_{i}$ для $1 \leq i \leq k$.

Пусть теперь $X$ - полупростая алгебраическая группа и $\lambda, \gamma, \mu$ - ее доминантные веса такие, что модули $T_{X}(\lambda)=\mu|\lambda| \mu$ и $T_{X}(\gamma)=\mu|\gamma| \mu$ унисериальны. Следуя [18], через $\Delta(\lambda ; \gamma)$ будем обозначать неразложимый модуль вида $\mu|(\lambda \oplus \gamma)| \mu$, точное определение которого приводится в [18, разд. 9.1].

Предложение 1 [17, теорема 2]. Пусть (во введенных выше обозначениях) $L=R^{F}-u c$ ключительная группа лиева типа, изоморфная группе $E_{8}(q)$. Предположим, что $G$ - группа со свойством $L \leq G \leq \operatorname{Aut}(L)$ и $M-$ максимальная подгруппа группь $G$. Тогда либо $F^{*}(M)$ является простой группой, либо выполняется одно из следующих утверждений.

I. $M=N_{G}\left(U^{F}\right)$, где $U$ является либо параболической подгруппой группь $R$, либо редуктивной подгруппой максимального ранга группы $R$.

II. $M=N_{G}(E)$, где $E$ является элементарной абелевой подгруппой из [4, теорема 1(II)].

III. $M=C_{G}(\tau)$, где $\tau-$ полевой автоморфизм простого порядка группы $L$.

IV. $p>5$ и либо $F^{*}(M) \cong \operatorname{Alt}_{5} \times \operatorname{Alt}_{6}(M-$ подгруппа Боровика группы $G)$, либо $F^{*}(M) \cong$ $\operatorname{Alt}_{5} \times L_{2}(q)$.

V. Группа $F^{*}(M)$ изоморфна одной из следующих групп (см. [17, табл. 3]):

a) $F^{*}(M) \cong L_{2}(q) \times L_{3}^{\epsilon}(q), p>3 ; \quad$ b) $F^{*}(M) \cong G_{2}(q) \times F_{4}(q)$;

c) $F^{*}(M) \cong L_{2}(q) \times G_{2}(q) \times G_{2}(q), p>2, q>3$; $\quad$ d) $F^{*}(M) \cong L_{2}(q) \times G_{2}\left(q^{2}\right), p>2, q>3$. 
Предложение 2. Пусть $G$ - почти простая примитивная группа подстановок на конечном множестве $X$ и $T:=\operatorname{soc}(G)$. Предположим, что $T \cong E_{8}(q)$, где $q-$ степень простого числа. Если для группы подстановок Т выполняется свойство $(\mathbf{P r})$, то свойство $(\mathbf{P r})$ выполняется и для группы подстановок $G$.

Д о к а з а т е л ь с т в о. Предположим, что для группы подстановок $T$ выполняется свойство (Pr). Пусть $x, y-$ различные элементы из $X$ и $G_{x, y} \unlhd G_{x}$. Покажем, что $G_{x, y}=1$.

Рассмотрим естественный гомоморфизм $\phi$ группы $G$ в факторгруппу $G / T$. Тогда $G_{x, y} \cap$ $\operatorname{ker} \phi=G_{x, y} \cap T=T_{x, y}=1$. Таким образом, группа $G_{x, y}$ изоморфно вкладывается в группу $G / T$ и, следовательно, в группу $\operatorname{Out}\left(E_{8}(q)\right) \cong \operatorname{Aut}\left(\mathbb{F}_{q}\right)$. Поскольку в силу [11, предложение 8] имеем $F\left(G_{x, y}\right)=1$, то $G_{x, y}=1$. Таким образом, для группы подстановок $G$ выполняется свойство $(\mathbf{P r})$.

Предложение 3. Пусть $G$ - примитивная группа подстановок на конечном множестве X. Предположим, что $G \leq H \mathrm{wr} S_{m}$ - группа типа III(b) и $H$ - примитивная почти простал группа с иоколем, изоморфным $E_{8}(q)$, где $q$ - степень простого числа. Если для группы подстановок $Н$ выполняется свойство (Pr), то для группы подстановок $G$ выполняется свойство $(\mathbf{P r})$.

Д о к а з а т е л ь с т в о. Справедливость предложения следует из [11, предложение 18] и того факта, что группа автоморфизмов группы $\operatorname{soc}(H)$ не содержит графовых автоморфизмов.

\section{3. Доказательство теорем 1 и 2}

В силу предложений 2 и 3 для доказательства теорем 1 и 2 достаточно показать, что если $G \cong E_{8}(q)-$ примитивная группа подстановок на конечном множестве $X$, то для группы подстановок $G$ выполняется свойство (Pr). Поэтому далее будем считать, что $G=L=R^{F}$. (Здесь и далее используются обозначения, введенные выше.)

Пусть $x \in X$. По предложению 1 либо $F^{*}\left(G_{x}\right)$ является простой группой, либо для $G_{x}$ выполняется одно из утверждений $\mathrm{I}-\mathrm{V}$ предложения 1.

Если $F^{*}\left(G_{x}\right)$ является простой группой, то $G_{x}$ является почти простой группой. Поэтому $G_{x} / \operatorname{soc}\left(G_{x}\right)$ является (согласно "гипотезе Шрейера") разрешимой группой и в силу [11, предложение 20] для группы подстановок $G$ выполняется свойство (Pr).

Таким образом, не теряя общности, далее будем считать, что для $G_{x}$ выполняется одна из возможностей $\mathrm{I}-\mathrm{V}$ предложения 1 . Кроме того, при рассмотрении случаев 1.1-1.8 будем учитывать, что $F\left(G_{x, y}\right)=1$ (см. [12, предложение 1$]$ ).

Случай 1. Для $G_{x}$ выполняется утверждение I предложения 1 , т. е. $G_{x}=N_{G}\left(U^{F}\right)$, где $U$ является либо параболической подгруппой группы $R$, либо редуктивной подгруппой максимального ранга группы $R$.

Пусть $U$ является параболической подгруппой группы $R$. Тогда $G_{x}$ является параболической подгруппой группы $R^{F}$ и, следовательно, $C_{G_{x}}\left(O_{r}\left(G_{x}\right)\right) \leq O_{r}\left(G_{x}\right)$ для некоторого простого числа $r$. Предположим, что $G_{x, y} \unlhd G_{x}$. Тогда $G_{x, y} \cap O_{r}\left(G_{x}\right)=1$ [11, предложение 8], $\left[O_{r}\left(G_{x}\right), G_{x, y}\right]=1$ и $G_{x, y} \leq C_{G_{x}}\left(O_{r}\left(G_{x}\right)\right) \leq O_{r}\left(G_{x}\right)$. Таким образом, $G_{x, y}=1$ и для $G$ выполняется свойство $(\mathrm{Pr})$.

Пусть $U$ является редуктивной подгруппой максимального ранга группы $R$. Все редуктивные подгруппы максимального ранга конечных исключительных групп лиева типа перечислены в [16, табл. 5.1]. Без ограничения общности можно предполагать (см. [20, теоремы 29.1, 29.5]), что $G_{x}=M=H^{F}$, где $H$ - замкнутая $F$-инвариантная подгруппа группы $R$, максимальная среди $F$-инвариантных замкнутых подгрупп группы $R$, имеющих положительную размерность. Далее будет показано выполнение свойства $(\mathbf{P r})$ для группы $G$ при этом условии. 
Пусть $y \in X \backslash\{x\}$ и $G_{x, y} \unlhd G_{x}$. Проверим, что $G_{x, y}=1$. Поскольку из $G_{x, y} \unlhd G_{y}$ следует $G_{x, y}=1$, то далее будем предполагать, что $G_{x, y} \not G_{y}$. Покажем, что в каждом из возникающих здесь случаев 1.1-1.8 (см. [16, табл. 5.1]), это приводит к противоречию.

Случай 1.1. $G_{x} \cong d .\left(L_{2}(q) \times E_{7}(q)\right) . d$, где $d=(2, q-1)$.

Если $d=2$, то $Z\left(G_{x}\right) \neq 1$ и получаем противоречие с [12, лемма 1$]$. Поэтому имеем $d=1$ и $p=2$. Из $[22,(1.8)]$ следует, что $H=A_{1} \circ E_{7}\left(A_{1}, E_{7}\right.$ - полупростые алгебраические группы типов $A_{1}(K)$ и $E_{7}(K)$ соответственно), при этом векторное пространство $\mathcal{L}(R)$, рассматриваемое как $K H$-модуль, имеет (см. $[22,(1.8)]$ в в указанных в разделе 2 обозначениях следующее разложение:

$$
\mathcal{L}\left(A_{1}\right) \oplus \mathcal{L}\left(E_{7}\right) \oplus(1 \otimes 0000001) .
$$

Пусть $A, B$ - подгруппы группы $G_{x}$ такие, что $A=A_{1}^{F} \cong L_{2}(q), B=E_{7}^{F} \cong E_{7}(q)$ и $G_{x}=A \times B$. Поскольку $G_{x, y} \unlhd G_{x}$, то $\operatorname{soc}\left(G_{x, y}\right) \in\{1, A, B, A \times B\}$. В случае, когда $\operatorname{soc}\left(G_{x, y}\right) \unlhd G_{y}$, очевидно, что для группы $G$ выполняется свойство ( $\left.\mathrm{Pr}\right)$. Поэтому далее рассмотрим оставшийся случай, когда $\operatorname{soc}\left(G_{x, y}\right)=A$ и $A$ сопряжена в $G$ с некоторой диагональной подгруппой $D$ группы $A \times B$.

Сравнение количеств одномерных инвариантных подпространств у $A$ и у $D$ на $\mathcal{L}\left(A_{1}\right) \oplus$ $\mathcal{L}\left(E_{7}\right)$ приводит (в силу сопряженности $A$ и $D$ в $\left.G\right)$ к тому, что $1 \otimes 0000001$ как $K D$-модуль имеет прямое одномерное слагаемое. Тогда в силу [1, теорема 2.1] число $p$ не делит 2 ; противоречие. Таким образом, для группы $G$ выполняется свойство (Pr).

Случай 1.2. $G_{x} \cong e .\left(L_{3}^{\epsilon}(q) \times E_{6}^{\epsilon}(q)\right) . e .2$, где $\epsilon \in\{-1,+1\}$ и $e=(3, q-\epsilon)$.

Если $e=3$, то $Z\left(G_{x}\right) \neq 1$ и получаем противоречие с [12, лемма 1]. Поэтому имеем $e=1$. Из $[22,(1.8)]$ следует, что $H=A_{2} \circ E_{6}\left(A_{2}, E_{6}\right.$ - полупростые алгебраические группы типов $A_{2}(K)$ и $E_{6}(K)$ соответственно), при этом векторное пространство $\mathcal{L}(R)$, рассматриваемое как $K H$-модуль, имеет (см. $[22,(1.8)])$ в указанных в разд. 2 обозначениях следующее разложение:

$$
\mathcal{L}\left(A_{2}\right) \oplus \mathcal{L}\left(E_{6}\right) \oplus(000001 \otimes 10) \oplus(100000 \otimes 01) .
$$

Пусть $A, B$ - подгруппы группы $G_{x}$ такие, что $A=A_{2}^{F} \cong L_{3}^{\epsilon}(q), B=E_{6}^{F} \cong E_{6}^{\epsilon}(q)$ и $G_{x}=A \times B$. Поскольку $G_{x, y} \unlhd G_{x}$, то $\operatorname{soc}\left(G_{x, y}\right) \in\{1, A, B, A \times B\}$. В случае, когда $\operatorname{soc}\left(G_{x, y}\right) \unlhd G_{y}$, очевидно, что для группы $G$ выполняется свойство $(\mathbf{P r})$. Поэтому далее рассмотрим оставшийся случай, когда $\operatorname{soc}\left(G_{x, y}\right)=A$ и $A$ сопряжена в $G$ с некоторой диагональной подгруппой $D$ группы $A \times B$.

Группа $A$ действует тривиально на $\mathcal{L}\left(E_{6}\right)$, и $(000001 \otimes 10) \oplus(100000 \otimes 01)$ как $K A$-модуль распадается в сумму трехмерных модулей, изоморфных модулю 10 (см. [19]). Тогда $000001 \otimes 10$ и $100000 \otimes 01$ как $K D$-модули есть прямая сумма одномерных и трехмерных модулей, изоморфных 10. При этом, по крайней мере, один из этих двух модулей должен содержать в качестве прямого слагаемого одномерный тривиальный модуль. Ниже рассуждения будут проведены для модуля $000001 \otimes 10$, для второго модуля $100000 \otimes 01$ рассуждения повторяются практически дословно.

Итак, предположим, что $K D$-модуль $000001 \otimes 10$ есть прямая сумма тривиальных одномерных модулей и трехмерных модулей, изоморфных 10. Пусть 000001 как $K D$-модуль есть прямая сумма неразложимых слагаемых $\oplus_{i} a_{i}$. Поскольку $K D$-модуль $000001 \otimes 10$ содержит в качестве прямого слагаемого одномерный тривиальный модуль, то по [10, разд. 2.2] имеем $a_{i} \cong 10^{*}$ и по $[8$, лемма $2.2(\mathrm{ii})]$ имеем $a_{i} \otimes 10 \cong W_{0} \otimes W_{1}$, где $\operatorname{dim}\left(W_{0}\right)=1, \operatorname{dim}\left(W_{1}\right)=8$ и $W_{1}$ не содержит прямых одномерных слагаемых. Но тогда $W_{1}$ должен раскладываться в прямую сумму трехмерных неразложимых модулей; противоречие.

Таким образом, для группы $G$ выполняется свойство $(\mathrm{Pr})$.

Случай 1.3. $G_{x} \cong g \cdot\left(L_{5}^{\epsilon}(q)\right)^{2} . g .4$, где $g=(5, q-\epsilon), \epsilon \in\{-1,+1\}$.

Если $g=5$, то $Z\left(G_{x}\right) \neq 1$ и получаем противоречие с [12, лемма 1]. Поэтому имеем $g=1$. Из $[22,(1.8)]$ следует, что $H=A_{4}^{(1)} \circ A_{4}^{(2)}\left(A_{4}^{(i)}-\right.$ полупростая алгебраическая группа типа $A_{4}(K)$, 
$i \in\{1,2\})$, при этом векторное пространство $\mathcal{L}(R)$, рассматриваемое как $K H$-модуль, имеет (см. $[22,(1.8)])$ в указанных в разд. 2 обозначениях следующее разложение:

$$
\mathcal{L}\left(A_{4}^{(1)}\right) \oplus \mathcal{L}\left(A_{4}^{(2)}\right) \oplus(0010 \otimes 1000) \oplus(0100 \otimes 0001) \oplus(1000 \otimes 0100) \oplus(0001 \otimes 0010) .
$$

Пусть $A, B$ - подгруппы группы $G_{x}$ такие, что $A=\left(A_{4}^{(1)}\right)^{F} \cong L_{5}^{\epsilon}(q), B=\left(A_{4}^{(2)}\right)^{F} \cong L_{5}^{\epsilon}(q)$ и $G_{x}=A \times B$. Поскольку $G_{x, y} \unlhd G_{x}$, то $\operatorname{soc}\left(G_{x, y}\right) \in\{1, A, B, A \times B\}$. В случае, когда $\operatorname{soc}\left(G_{x, y}\right) \unlhd G_{y}$, очевидно, что для группы $G$ выполняется свойство $(\mathrm{Pr})$. Поэтому далее рассмотрим оставшийся случай, когда $\operatorname{soc}\left(G_{x, y}\right)=A$ и $A$ сопряжена в $G$ с некоторой диагональной подгруппой $D$ группы $A \times B$.

Группа $A$ действует тривиально на $\mathcal{L}(B)$, и

$$
(0010 \otimes 1000) \oplus(0100 \otimes 0001) \oplus(1000 \otimes 0100) \oplus(0001 \otimes 0010)
$$

как $K A$-модуль распадается в сумму пятимерных и десятимерных модулей (см. [19]). В частности, пространство $\mathcal{L}(R)$ как $K A$-модуль имеет ровно одно инвариантное подпространство размерности 24 . При этом пространство $\mathcal{L}(R)$ как $K D$-модуль имеет два инвариантных подпространства размерности $24(\mathcal{L}(A)$ и $\mathcal{L}(B))$; противоречие.

Таким образом, для группы $G$ выполняется свойство $(\mathbf{P r})$.

Случай 1.4. $G_{x} \cong d^{2} \cdot\left(P \Omega_{8}^{+}(q)\right)^{2} \cdot d^{2} \cdot\left(S_{3} \times 2\right)$, где $d=(2, q-1)$.

Из $[22,(1.8)]$ следует, что $H=D_{4}^{(1)} \circ D_{4}^{(2)}\left(D_{4}^{(i)}-\right.$ полупростая алгебраическая группа типа $\left.D_{4}(K), i \in\{1,2\}\right)$, при этом векторное пространство $\mathcal{L}(R)$, рассматриваемое как $K H$-модуль, имеет (см. $[22,(1.8)])$ в указанных в разд. 2 обозначениях следующее разложение:

$$
\mathcal{L}\left(D_{4}^{(1)}\right) \oplus \mathcal{L}\left(D_{4}^{(2)}\right) \oplus(0010 \otimes 0010) \oplus(0001 \otimes 0001) \oplus(1000 \otimes 1000) .
$$

Поскольку $G_{x, y} \unlhd G_{x}$, то из [12, предложение 1] имеем $F\left(G_{x, y}\right)=1$ и $\operatorname{soc}\left(G_{x, y}\right) \in\{1, A, B, A \times$ $B\}$, где $A, B$ - подгруппы группы $G_{x}$ такие, что $A=\left(D_{4}^{(1)}\right)^{F} \cong P \Omega_{8}^{+}(q), B=\left(D_{4}^{(2)}\right)^{F} \cong P \Omega_{8}^{+}(q)$. В случае, когда $\operatorname{soc}\left(G_{x, y}\right) \unlhd G_{y}$, очевидно, что для группы $G$ выполняется свойство (Pr). Поэтому далее рассмотрим оставшийся случай, когда $\operatorname{soc}\left(G_{x, y}\right)=A$ и $A$ сопряжена в $G$ с некоторой диагональной подгруппой $D$ группы $A \times B$.

Группа $A$ действует тривиально на $\mathcal{L}(B)$, и

$$
(0010 \otimes 0010) \oplus(0001 \otimes 0001) \oplus(1000 \otimes 1000)
$$

как $K A$-модуль распадается в сумму восьмимерных модулей (см. [19]). В частности, пространство $\mathcal{L}(R)$ как $K A$-модуль имеет ровно одно инвариантное подпространство размерности 28 . При этом пространство $\mathcal{L}(R)$ как $K D$-модуль имеет два инвариантных подпространства размерности $28(\mathcal{L}(A)$ и $\mathcal{L}(B))$; противоречие.

Таким образом, для группы $G$ выполняется свойство $(\mathrm{Pr})$.

Случай 1.5. $G_{x} \cong\left({ }^{3} D_{4}(q)\right)^{2} .6$.

Из [16, лемма 2.5] следует, что $G_{x}$ действует транзитивно на прямых множителях группы $\operatorname{soc}\left(G_{x}\right)$, изоморфных ${ }^{3} D_{4}(q)$. Поэтому $F^{*}\left(G_{x, y}\right) \cong\left({ }^{3} D_{4}(q)\right)^{2}$ и для группы $G$ выполняется свойство (Pr).

Случай 1.6. $G_{x} \cong e^{2} \cdot\left(L_{3}^{\epsilon}(q)\right)^{4} \cdot e^{2} \cdot G L_{2}(3)$, где $\epsilon \in\{-1,+1\}$ и $e=(3, q-\epsilon)$.

Из [16, лемма 2.5] следует, что $G_{x}$ действует транзитивно на прямых множителях группы $\operatorname{soc}\left(G_{x}\right)$, изоморфных $L_{3}^{\epsilon}(q)$. Поэтому $F^{*}\left(G_{x, y}\right) \cong\left(L_{3}^{\epsilon}(q)\right)^{4}$ и для группы $G$ выполняется свойство (Pr).

Случай 1.7. $G_{x} \cong\left(U_{3}\left(q^{2}\right)\right)^{2} .8$.

Из [16, лемма 2.5] следует, что $G_{x}$ действует транзитивно на прямых множителях группы $\operatorname{soc}\left(G_{x}\right)$, изоморфных $U^{3}\left(q^{2}\right)$. Поэтому $F^{*}\left(G_{x, y}\right) \cong\left(U_{3}\left(q^{2}\right)\right)^{2}$ и для группы $G$ выполняется свойство (Pr). 
Случай 1.8. $G_{x} \cong d^{4} \cdot\left(L_{2}(q)\right)^{8} \cdot d^{4} \cdot A G L_{3}(2)$, где $q>2$ и $d=(2, q-1)$.

Если $q=3$, то для группы $G$ выполняется свойство (Pr) по [12, предложение 1]. Далее считаем, что $q>3$.

Из [16, лемма 2.5] следует, что $G_{x}$ действует транзитивно на прямых множителях группы $\operatorname{soc}\left(G_{x}\right)$, изоморфных $L_{2}(q)$. Поэтому $F^{*}\left(G_{x, y}\right) \cong\left(L_{2}(q)\right)^{8}$ и для группы $G$ выполняется свойство (Pr).

Таким образом, случай 1 полностью рассмотрен.

Случай 2. Для $G_{x}$ выполняется утверждение II предложения 1.

Согласно [4, теорема $1(\mathrm{II})]$ группа $G_{x}$ в этом случае имеет вид $A . K . B$, где $A, B-$ paзрешимые группы и $K$ - простая неабелева группа. Следовательно, по [11, предложение 9] для группы $G$ выполняется свойство (Pr).

Случай 3. Для $G_{x}$ выполняется утверждение III предложения 1.

Имеем $G_{x}=C_{G}(\tau)$, где $\tau$ - полевой автоморфизм простого порядка группы $G$. Из доказательства $[17$, лемма 3.1$]$ следует, что в этом случае либо $F^{*}\left(G_{x}\right)$ является простой группой, либо $F\left(G_{x}\right) \neq 1$ и $F\left(G_{x}\right) \leq Z\left(G_{x}\right)$. Если $F^{*}\left(G_{x}\right)$ является простой группой, то по [11, предложение 20] для группы $G$ выполняется свойство $(\operatorname{Pr})$. Если $F\left(G_{x}\right) \neq 1$ и $F\left(G_{x}\right) \leq Z\left(G_{x}\right)$, то $Z\left(G_{x}\right) \neq 1$; противоречие с [12, лемма 1$]$.

Случай 4. Для $G_{x}$ выполняется утверждение IV предложения 1, и группа $G_{x}$ не является подгруппой Боровика группы $G$ (то есть $\left.F^{*}\left(G_{x}\right) \supsetneqq \operatorname{Alt}_{5} \times \operatorname{Alt}_{6}\right)$. Тогда имеем $p>5$ и $F^{*}\left(G_{x}\right) \cong$ $\operatorname{Alt}_{5} \times L_{2}(q)$.

В силу $\left[17\right.$, лемма 1.5] получаем $G_{x}=C_{G}(Y) \times Y^{F}$, где $Y \leq R$ имеет тип $A_{1}$ и $C_{G}(Y) \cong \mathrm{Sym}_{5}$. Покажем, что для группы $G$ выполняется свойство (Pr).

Пусть $y \in X \backslash\{x\}$ и $G_{x, y} \unlhd G_{x}$. Проверим, что $G_{x, y}=1$. Поскольку из $G_{x, y} \unlhd G_{y}$ следует $G_{x, y}=1$, то далее будем предполагать, что $G_{x, y} \notin G_{y}$. Тогда $\operatorname{soc}\left(G_{x, y}\right)=C_{G}(Y)^{\prime}$, и группа $C_{G}(Y)^{\prime}$ сопряжена в $G$ с некоторой диагональной подгруппой $D$ группы $C_{G}(Y) \times Y^{F}$. Пусть $Z$ - проекция группы $D$ на группу $Y^{F}, D \cong Z \cong \operatorname{Alt}_{5}$. Тогда $Z \times C_{G}(Z)$ - цоколь некоторой подгруппы Боровика группы $G$ (см. $[17$, лемма 1.5$])$, и $D$ является некоторой диагональной подгруппой группы $Z \times C_{G}(Z)$. Таким образом, группа $D$ сопряжена в $G$ с некоторой $\mathrm{Alt}_{5-}$ подгруппой $B$ из $\mathrm{Sym}_{6}$-подгруппы некоторой подгруппы Боровика группы $G$. Но 3 -элементы групп $D$ и $B$ имеют неизоморфные централизаторы в группе $G$ (см. [2]); противоречие.

Случай 5. Для $G_{x}$ выполняется утверждение $\mathrm{V}$ предложения 1.

Пусть $y \in X \backslash\{x\}$ и $G_{x, y} \unlhd G_{x}$. Проверим, что $G_{x, y}=1$. Поскольку из $G_{x, y} \unlhd G_{y}$ следует $G_{x, y}=1$, то далее будем предполагать, что $G_{x, y} \not G_{y}$. Покажем, что это приводит к противоречию.

Так как для $G_{x}$ выполняется утверждение $\mathrm{V}$ предложения 1 , то $\operatorname{soc}\left(G_{x}\right)=F^{*}\left(G_{x}\right)$ имеет вид $A \times B$ или $A \times B \times B$, где $A$ и $B$ - простые неабелевы группы, $|A|<|B|$. Из строения $F^{*}\left(G_{x}\right)$ следует, что $G_{x}$ имеет вид $\operatorname{soc}\left(G_{x}\right) . W$, где $W$ - разрешимая группа. Таким образом, $\operatorname{soc}\left(G_{x, y}\right) \unlhd G_{x}, \operatorname{soc}\left(G_{x, y}\right) \unlhd \operatorname{soc}\left(G_{x}\right)$.

Покажем, что $\operatorname{soc}\left(G_{x, y}\right) \nsupseteq \operatorname{soc}\left(G_{y}\right)$. Предоположим противное. Тогда

$$
\operatorname{soc}\left(G_{x, y}\right) \unlhd\left\langle G_{x}, \operatorname{soc}\left(G_{y}\right)\right\rangle .
$$

Если $\left\langle G_{x}, \operatorname{soc}\left(G_{y}\right)\right\rangle=G$, то $\operatorname{soc}\left(G_{x, y}\right) \unlhd G$; противоречие. Поэтому $\operatorname{soc}\left(G_{y}\right) \leq G_{x}$, и в силу строения $F^{*}\left(G_{x}\right)$ имеем $\operatorname{soc}\left(G_{y}\right)=\operatorname{soc}\left(G_{x}\right)$ и $\operatorname{soc}\left(G_{x}\right) \unlhd G$; противоречие. Таким образом, $\operatorname{soc}\left(G_{x, y}\right) \not \operatorname{soc}\left(G_{y}\right)$.

Итак, $\operatorname{soc}\left(G_{x}\right)$ имеет вид $A \times B$ или $A \times B \times B$, где $A$ и $B$ - простые неабелевы группы, $|A|<|B|$. При этом $\operatorname{soc}\left(G_{x, y}\right) \unlhd \operatorname{soc}\left(G_{x}\right)$ и $\operatorname{soc}\left(G_{x, y}\right) \nsupseteq \operatorname{soc}\left(G_{y}\right)$. Другими словами, $\operatorname{soc}\left(G_{x, y}\right)^{g} \nsupseteq$ $\operatorname{soc}\left(G_{x}\right)$ для любого $g \in G$ со свойством $g(x)=y$.

Предположим, что $\operatorname{soc}\left(G_{x}\right)$ имеет вид $A \times B$. Тогда $\operatorname{soc}\left(G_{x, y}\right) \in\{1, A, B, A \times B\}$. Поскольку $\operatorname{soc}\left(G_{x, y}\right) \not \operatorname{soc}\left(G_{y}\right)$, имеем $\operatorname{soc}\left(G_{x, y}\right)=A$ и $\operatorname{soc}\left(G_{x, y}\right)^{g}$, где $g \in G$ и $g(x)=y$ - диагональная подгруппа $D$ в группе $A \times B$, сопряженная с $A$ в $G$. 
Предположим, что $\operatorname{soc}\left(G_{x}\right)$ имеет вид $A \times B \times B$. Тогда, аналогично, получаем, что либо в группе $A \times B$ есть диагональная подгруппа $D$, сопряженная в $G$ с $A$, либо в группе $B \times B$ есть диагональная подгруппа $D$, сопряженная в $G$ с $B$.

Покажем, что в каждом из возникающих здесь случаев 5.1-5.4 это приводит к противоречию.

Случай 5.1. $A \cong L_{2}(q), B \cong L_{3}^{\epsilon}(q)$, где $p \geq 5$.

Из [18] следует, что $H=A_{1} \circ A_{2}\left(A_{1}, A_{2}\right.$ - полупростые алгебраические группы типов $A_{1}(K)$ и $A_{2}(K)$ соответственно).

Если $p \geq 7$, то из [18, табл. (10.1)] следует, что векторное пространство $\mathcal{L}(R)$, рассматриваемое как $K H$-модуль, имеет следующее разложение:

$$
(2 \otimes 22) \oplus(6 \otimes 11) \oplus(4 \otimes 30) \oplus(4 \otimes 03) \oplus(2 \otimes 00) \oplus(0 \otimes 11) .
$$

Как $K A$-модуль $\mathcal{L}(R)$ состоит из прямой суммы простых неприводимых модулей $0,2,4,6$; противоречие с тем, что подпространство $6 \otimes 11$ пространства $\mathcal{L}(R)$ как $K D$-модуль по [6, лемма 3.1] содержит модуль $T(r)$, где $r \geq 7$.

Если $p=5$, то из $[18$, табл. (10.1)] следует, что векторное пространство $\mathcal{L}(R)$, рассматриваемое как $K H$-модуль, имеет следующее разложение:

$$
\Delta(2 \otimes 22 ; 6 \otimes 11) \oplus(4 \otimes 30) \oplus(4 \otimes 03) \oplus(2 \otimes 00) \oplus(0 \otimes 11) .
$$

Секции прямых слагаемых $\mathcal{L}(R)$ как $K A$-модуля - это модули $0,2,4,6$. Аналогично случаю $p \geq 7$ применение [6, лемма 3.1] приводит к противоречию.

Таким образом, для группы $G$ выполняется свойство $(\mathrm{Pr})$.

Случай 5.2. $A \cong G_{2}(q), B \cong F_{4}(q)$.

Из [18] следует, что $H=G_{2} \circ F_{4}\left(G_{2}, F_{4}\right.$ - полупростые алгебраические группы типов $G_{2}(K)$ и $F_{4}(K)$ соответственно).

Если $p \geq 5$, то из [18, табл. (10.1)] следует, что векторное пространство $\mathcal{L}(R)$, рассматриваемое как $K H$-модуль, имеет следующее разложение: $(01 \otimes 0000) \oplus(00 \otimes 1000) \oplus(10 \otimes 0001)$.

Из [19] следует, что $\mathcal{L}(R)$ как $K A$-модуль состоит из прямой суммы неприводимых модулей размерностей 1,7 и 14 . Следовательно, $10 \otimes 0001$ как $K D$-модуль раскладывается в прямую сумму неприводимых модулей размерностей 1 и 14 . Противоречие следует из [20, предложение $15.12 ; 19]$.

Если $p=3$, то из $[18$, табл. (10.1)] следует, что векторное пространство $\mathcal{L}(R)$, рассматриваемое как $K H$-модуль, имеет следующее разложение: $\Delta(10 \otimes 0001 ; 01 \otimes 0000) \oplus(00 \otimes 1000)$. Таким образом, неприводимые прямые слагаемые $\mathcal{L}(R)$ как $K A$-модуля - это $T(01), 10$ и 00. Противоречие следует из [20, предложение $15.12 ; 19]$.

Если $p=2$, то из [18, табл. (10.1)] следует, что векторное пространство $\mathcal{L}(R)$, рассматриваемое как $K H$-модуль, имеет следующее разложение: $\Delta(10 \otimes 0001 ; 00 \otimes 1000) \oplus(01 \otimes 0000)$. Таким образом, $\mathcal{L}(R)$ как $K A$-модуль содержит в качестве прямого неприводимого слагаемого $T(10)^{24}$ (см. [18, лемма 9.1.5]); противоречие.

Таким образом, для группы $G$ выполняется свойство $(\mathbf{P r})$.

Случай 5.3. $\operatorname{soc}\left(G_{x}\right)$ имеет вид $A \times B_{1} \times B_{2}, A \cong L_{2}(q), B_{1} \cong B_{2} \cong G_{2}(q)$, где $p \geq 3$ и $q \geq 5$.

Из [18] следует, что $H=A_{1} \circ G_{2}^{(1)} \circ G_{2}^{(2)}\left(A_{1}, G_{2}^{(i)}\right.$ - полупростые алгебраические группы типов $A_{1}(K)$ и $G_{2}(K)$ соответственно, $\left.i \in\{1,2\}\right)$.

Если $p \geq 5$, то из [18, табл. (10.1)] следует, что векторное пространство $\mathcal{L}(R)$, рассматриваемое как $K H$-модуль, имеет следующее разложение:

$(2 \otimes 10 \otimes 10) \oplus(4 \otimes 10 \otimes 00) \oplus(4 \otimes 00 \otimes 10) \oplus(2 \otimes 00 \otimes 00) \oplus(0 \otimes 01 \otimes 00) \oplus(0 \otimes 00 \otimes 01)$.

Напомним, что если $\operatorname{soc}\left(G_{x}\right)$ имеет вид $A \times B \times B$, то либо в группе $A \times B$ есть диагональная подгруппа $D \cong A$, сопряженная в $G$ с $A$, либо в группе $B \times B$ есть диагональная подгруппа 
$D \cong B$, сопряженная в $G$ с $B$. Если в группе $A \times B$ есть диагональная подгруппа $D \cong A$, сопряженная в $G$ с $A$, то получаем противоречие аналогично случаю 5.1. Если в группе $B \times B$ есть диагональная подгруппа $D \cong B$, сопряженная в $G$ с $B$, то получаем противоречие на основе [20, предложение $15.12 ; 19]$.

Если $p=3$, то из $[18$, табл. (10.1)] следует, что векторное пространство $\mathcal{L}(R)$, рассматриваемое как $K M$-модуль, имеет следующее разложение:

$(2 \otimes 10 \otimes 10) \oplus \Delta(4 \otimes 10 \otimes 00 ; 0 \otimes 01 \otimes 00) \oplus(2 \otimes 00 \otimes 00) \oplus \Delta(4 \otimes 00 \otimes 10 ; 0 \otimes 00 \otimes 01)$.

Аналогично случаю 5.1 применение [20, предложение 15.12; 19] приводит к противоречию.

Таким образом, для группы $G$ выполняется свойство $(\mathrm{Pr})$.

Случай 5.4. $A \cong L_{2}(q), B \cong G_{2}\left(q^{2}\right)$, где $p \geq 3$ и $q \geq 5$.

Из [18] следует, что $H=A_{1} \circ G_{2}^{(1)} \circ G_{2}^{(2)}\left(A_{1}, G_{2}^{(i)}-\right.$ полупростые алгебраические группы типов $A_{1}(K)$ и $G_{2}(K)$ соответственно, $\left.i \in\{1,2\}\right)$.

Если $p \geq 5$, то из $[18$, табл. (10.1)] следует, что векторное пространство $\mathcal{L}(R)$, рассматриваемое как $K H$-модуль, имеет следующее разложение (см. случай 5.3):

$(2 \otimes 10 \otimes 10) \oplus(4 \otimes 10 \otimes 00) \oplus(4 \otimes 00 \otimes 10) \oplus(2 \otimes 00 \otimes 00) \oplus(0 \otimes 01 \otimes 00) \oplus(0 \otimes 00 \otimes 01)$.

Как $K A$-модуль $\mathcal{L}(R)$ состоит из прямой суммы простых неприводимых модулей размерностей 1,3 и 5 . Следовательно, $\mathcal{L}(R)$ как $K D$-модуль должен быть вполне приводимым и раскладываться в прямую сумму подпространств размерностей 1,3 и 5 ; противоречие.

Если $p=3$, то из [18, табл. (10.1)] следует, что векторное пространство $\mathcal{L}(R)$, рассматриваемое как $K H$-модуль, имеет следующее разложение (см. случай 5.3):

$$
(2 \otimes 10 \otimes 10) \oplus \Delta(4 \otimes 10 \otimes 00 ; 0 \otimes 01 \otimes 00) \oplus(2 \otimes 00 \otimes 00) \oplus \Delta(4 \otimes 00 \otimes 10 ; 0 \otimes 00 \otimes 01) .
$$

Аналогично случаю $p \geq 5$, слагаемое $0 \otimes 01 \otimes 00$ дает секцию размерности 7 (см. [19]); противоречие.

Доказательство теорем 1 и 2 завершено.

\section{СПИСОК ЛИТЕРАТУРЫ}

1. Benson D., Carlson J. Nilpotent elements in the Green ring // J. Algebra. 1986. Vol. 104. P. 329-350. doi: 10.1016/0021-8693(86)90219-X .

2. Borovik A. V. A maximal subgroup in the simple finite group $E_{8}(q) / /$ Contemporary Mathematics (131). 1992. Part 1, P. 67-79. doi: 10.1090/conm/131.1.

3. Cameron P.J. Suborbits in transitive permutation groups // Combinatorics: Proc. NATO Advanced Study Inst. (Breukelen, 1974). Part 3: Combinatorial Group Theory. Amsterdam: Math. Centrum, 1974. P. 98-129. (Math. Centre Tracts; vol. 57).

4. Cohen A.M., Liebeck M.W., Saxl J., Seitz G.M. The local maximal subgroups of exceptional groups of Lie type, finite and algebraic// Proc. London Mat. Soc. (3). 1992. Vol. 64. P. 21-48. doi: $10.1112 / \mathrm{plms} / \mathrm{s} 3-64.1 .21$.

5. Conway J.H. et. al. Atlas of finite groups. Oxford: Clarendon Press, 1985. 252 p. ISBN: 0-19-853199-0.

6. Craven D.A. On tensor products of simple modules for simple groups // Algebras and Representation Theory. 2013. Vol. 16, iss. 2. P. 377-404. doi: 10.1007/s10468-011-9311-5.

7. Doty S., Henke A. Decomposition of tensor products of modular irreducibles for $\mathrm{SL}_{2} / /$ Q. J. Math. Algebra. 2005. Vol. 56, no. 2. P. 189-207. doi: 10.1093/qmath/hah027.

8. Feit W. The representation theory of finite groups. North-Holland: Elsevier, 1982. 501 p. (North-Holland Mathematical Library). ISBN: 978-0-444-86155-9.

9. Фомин А.Н. Свойства подорбит конечных примитивных групп подстановок // Теоретикогрупповые исследования: сб. науч. тр. / УрО АН СССР. Свердловск, 1990. С. 87-94.

10. Humphreys J.E. Modular representations of finite groups of Lie type Cambridge: Cambridge University Press, 2011. 206 p. doi: 10.1017/CBO9780511525940 . 
11. Коныгин А.В. О примитивных группах подстановок со стабилизатором двух точек, нормальным в стабилизаторе одной из них // Сиб. электрон. мат. изв. 2008. Т. 5. С. 387-406.

12. Коныгин А.В. К вопросу П. Камерона о примитивных группах подстановок со стабилизатором двух точек, нормальным в стабилизаторе одной из них // Тр. Ин-та математики и механики УрО PAH. 2013. T. 19, № 3. C. 187-198.

13. Knapp W. Primitive Permutationsgruppen mit einem Subkonstituenten, dessen Stabilisatorgruppe Fittingfrei ist // Arch. Math. 1974. Vol. 25. P. 472-475. doi: 10.1007/BF01238709.

14. Knapp W. Some problems of Wielandt revisited // J. Algebra. 2006. Vol. 302, no. 1. P. 167-185. doi: $10.1016 /$ j.jalgebra.2005.09.013 .

15. Liebeck M.W., Praeger Ch.E., Saxl J. On the O'Nan-Scott theorem for finite primitive permutation groups // J. Austral. Math. Soc. Ser. A. 1988. Vol. 44, no. 3. P. 389-396. doi: $10.1017 /$ S144678870003216X.

16. Liebeck M.W., Saxl J., Seitz G.M. Subgroups of maximal rank in finite exceptional groups of Lie type // Proc. London Math. Soc. (3). 1992. Vol. 65, no. 2. P. 297-325. doi: 10.1112/plms/s3-65.2.297.

17. Liebeck M.W., Seitz G.M. Maximal subgroups of exceptional groups of Lie type, finite and algebraic // Geom. Dedicata. 1990. Vol. 35, no. 1-3. P. 353-387. doi: 10.1007/BF00147353.

18. Liebeck M.W., Seitz G.M. The maximal subgroups of positive dimension in exceptional algebraic groups. Providence: AMS, 2004. 227 p. ( Mem. Amer. Math. Soc.; vol. 169, no 802.) ISBN: 0-8218-3482-7.

19. Lubeck F. Small degree representations of finite Chevalley groups in defining characteristic // LMS J. Comput. Math. 2001. No. 4. P. 135-169. doi: 10.1112/S1461157000000838.

20. Malle G., Testerman D. Linear algebraic groups and finite groups of Lie type. Cambridge: Cambridge Univ. Press, 2011. 309 p. doi: 10.1017/CBO9780511994777.

21. Reitz H.L. On primitive groups of odd order // Amer. J. Math. 1904. Vol. 26. P. 1-30. doi: $10.2307 / 2369903$.

22. Seitz G.M. Maximal subgroups of exceptional algebraic groups Providence: AMS, 1991. 197 p. (Mem. Amer. Math. Soc.; vol. 90, no. 441.) ISBN: 0821825046.

23. Unsolved problems in group theory. The Kourovka Notebook / eds. E.I. Khukhro, V.D. Mazurov: [eresource]. 248 p. Available at: ArXiv:1401.0300v13 [math.GR] June 2018.

24. Weiss M.J. On simply transitive groups // Bull. Amer. Math. Soc. 1934. Vol. 40. P. 401-405. doi: 10.1090/S0002-9904-1934-05871-3.

25. Wielandt H. Finite permutation groups. N Y: Acad. Press, 1964. 114 p. ISBN: 9781483258294.

Поступила 19.09.2019

После доработки 18.11.2019

Принята к публикации 25.11.2019

Коныгин Антон Владимирович

канд. физ.-мат. наук, науч. сотрудник

Институт математики и механики им. Н.Н. Красовского УрО РАН

г. Екатеринбург

e-mail: konygin@imm.uran.ru

\section{REFERENCES}

1. Benson D., Carlson J. Nilpotent elements in the Green ring. J. Algebra, 1986, vol. 104, no. 2, pp. 329-350. doi: 10.1016/0021-8693(86)90219-X .

2. Borovik A. V. A maximal subgroup in the simple finite group $E_{8}(q)$ Contemporary Mathematics (131), 1992, part 1, pp. 67-79. doi: 10.1090/conm/131.1.

3. Cameron P.J. Suborbits in transitive permutation groups. In: Combinatorics: Proc. NATO Advanced Study Inst. (Breukelen, 1974), Part 3: Combinatorial Group Theory, Math. Centre Tracts, vol. 57. Amsterdam: Math. Centrum, 1974, pp. 98-129.

4. Cohen A.M., Liebeck M.W., Saxl J., Seitz G.M. The local maximal subgroups of exceptional groups of Lie type, finite and algebraic. Proc. London Mat. Soc. (3), 1992, vol. 64, no. 1, pp. 21-48. doi: $10.1112 / \mathrm{plms} / \mathrm{s} 3-64.1 .21$.

5. Conway J.H. et. al. Atlas of finite groups. Oxford: Clarendon Press, 1985, 252 p. ISBN: 0-19-853199-0 .

6. Craven D.A. On tensor products of simple modules for simple groups. Algebras and Representation Theory, 2013, vol. 16, iss. 2, pp. 377-404. doi: 10.1007/s10468-011-9311-5. 
7. Doty S., Henke A. Decomposition of tensor products of modular irreducibles for $\mathrm{SL}_{2}$. Q.J. Math. Algebra, 2005, vol. 56, no. 2, pp. 189-207. doi: 10.1093/qmath/hah027.

8. Feit W. The representation theory of finite groups. North Holland: Elsevier, 1982, $501 \mathrm{p}$. ISBN: 978-0-444-86155-9.

9. Fomin A.N. Properties of suborbits of finite primitive permutation groups. In: Starostin A.I. (ed.), Group-theoretic studies, Collection of scientific works, Sverdlovsk: Ural'skoe Otdelenie AN SSSR Publ., 1990, pp. 87-94.

10. Humphreys J.E. Modular representations of finite groups of Lie type. Cambridge: Cambridge University Press, 2011, 206 p. doi: 10.1017/CBO9780511525940.

11. Konygin A.V. On primitive permutation groups with a stabilizer of two points normal in the stabilizer of one of them. Sib. Elektron. Mat. Izv., 2008, vol. 5, pp. 387-406 (in Russian).

12. Konygin A.V. On Cameron's question about primitive permutation groups with stabilizer of two points that is normal in the stabilizer of one of them. Proc. Steklov Inst. Math. (Suppl.), 2014, vol. 285, pp. 116-127.

13. Knapp W. Primitive Permutationsgruppen mit einem Subkonstituenten, dessen Stabilisatorgruppe Fittingfrei ist. Arch. Math., 1974, vol. 25, no. 1, pp. 472-475. doi: 10.1007/BF01238709.

14. Knapp W. Some problems of Wielandt revisited. J. Algebra, 2006, vol. 302, no. 1, pp. 167-185. doi: 10.1016/j.jalgebra.2005.09.013.

15. Liebeck M.W., Praeger Ch.E., Saxl J. On the O'Nan-Scott theorem for finite primitive permutation groups. J. Austral. Math. Soc. Ser. A, 1988, vol. 44, no. 3, pp. 389-396. doi: 10.1017/S144678870003216X .

16. Liebeck M.W., Saxl J., Seitz G.M. Subgroups of maximal rank in finite exceptional groups of Lie type. Proc. London Math. Soc. (3), 1992, vol. 65, no. 2, pp. 297-325. doi: 10.1112/plms/s3-65.2.297.

17. Liebeck M.W., Seitz G.M. Maximal subgroups of exceptional groups of Lie type, finite and algebraic. Geom. Dedicata, 1990, vol. 35, no. 1-3, pp. 353-387. doi: 10.1007/BF00147353.

18. Liebeck M.W., Seitz G.M. The maximal subgroups of positive dimension in exceptional algebraic groups. Mem. Amer. Math. Soc., vol. 169, no. 802. Providence: AMS, 2004, 227 p. ISBN: 0-8218-3482-7.

19. Lubeck F. Small degree representations of finite Chevalley groups in defining characteristic. LMS J. Comput. Math., 2001, vol. 4, pp. 135-169. doi: 10.1112/S1461157000000838.

20. Malle G., Testerman D. Linear algebraic groups and finite groups of Lie type. Cambridge: Cambridge University Press, 2011. 309 p. doi: 10.1017/CBO9780511994777.

21. Rietz H.L. On primitive groups of odd order. Amer. J. Math., 1904, vol. 26, no. 1, pp. 1-30. doi: $10.2307 / 2369903$.

22. Seitz G.M. Maximal subgroups of exceptional algebraic groups. Mem. Amer. Math. Soc., vol. 90, no. 441. Providence: AMS, 1991, 197 p. ISBN: 0821825046.

23. Unsolved problems in group theory. The Kourovka notebook, Khukhro E.I., Mazurov V.D. (eds), no. 19. Novosibirsk: Russian Academy of Sciences, Siberain Branch, Sobolev Institute of Mathematics, 2018, 248 p. Available at: ArXiv:1401.0300v13 [math.GR] June 2018.

24. Weiss M.J. On simply transitive groups. Bull. Amer. Math. Soc., 1934, vol. 40, no. 6, pp. 401-405. doi: 10.1090/S0002-9904-1934-05871-3.

25. Wielandt H. Finite permutation groups. N Y: Acad. Press, 1964, 114 p. ISBN: 9781483258294.

Received September 19, 2019

Revised November 18, 2019

Accepted November 25, 2019

Anton Vladimirovich Konygin, Cand. Sci. (Phys.-Math.), Krasovskii Institute of Mathematics and Mechanics of the Ural Branch of the Russian Academy of Sciences, Yekaterinburg, 620108 Russia e-mail: konygin@imm.uran.ru .

Cite this article as: A. V. Konygin. On primitive permutation groups with the stabilizer of two points normal in the stabilizer of one of them: The case when the socle is a power of a group $E_{8}(q)$, Trudy Instituta Matematiki i Mekhaniki URO RAN, 2019, vol. 25, no. 4, pp. 88-98. 